\title{
Estratégias para disseminar a Metodologia de Aprendizagem baseada em Projetos na Comunidade IFPB
}

\section{Strategies to Spread the PrBL Methodology Through IFPB Community}

\section{Francisco Petrônio Alencar de Medeiros, Instituto Federal da Paraiba}

Palavras chaves: Aprendizagem Ativa, Aprendizagem baseada em Projetos,

Keywords: Active Learning, Project Based Learning, IFPB, learning outcomes, experiences in Finland.

\begin{abstract}
$\Delta$ clear question related to the growing gap between the educational model still in force in Brazil, as being passive, content-based and teacher-centered, against the expectations of students of $Z$ and alpha generation, as well as the demands of XXI century labor market, which requires innovation, creativity, collaboration, critical thinking, leadership, maximization of social and cultural interactions, has led to high rates of demotivation and consequent school dropout of students. The intense experience of three months in Finland, as a result of the participation in the Teachers for the Future 26/2015 SETEC / MEC / CNPq program, provided the author with a vast and transforming learning reach, as well as the possibility of observing in practice how the areas of collaborative and blended learning as well as educational technologies, besides being possible to be applied, could be improved and expanded. Returning to Brazil, more specifically to the IFPB classrooms, aroused the interest in disseminating this knowledge and experiences to IFPB teachers and managers, using different strategies described in this article. The actions that have been coordinated in the IFPB are included in the teaching, research and extension triad. In teaching, the experience in Finland has provided a significant change in how the learning process is conducted in the courses that the author is responsible for, as well as the possibility of offering training to teachers, managers and students on active learning methodologies, collaborative and social tools and formative and diagnostic evaluation. In the research, there are two ongoing projects, one of which is the development of the PrBL Tool for planning, execution and evaluation of Project-Based Learning, and the second that seeks to analyze the use of projects by IFPB teachers from the perspective of 21st Century competences and the Project-Based Learning premises. In the scope of extension, a project is underway for the construction and strengthening of a Collaborative Network of 21st Century Educators,
\end{abstract}


composed of teachers and managers of federal institutions and NGOs. All the ongoing actions are results of IFPB's calls and have shown significant results so far. The funding raised in the calls for proposals will promote scientific exchange through the presentation of papers at international conferences, as well as in the implementation of partnerships between Brazil and Finland researchers and practitioners for the dissemination of the educational experiences and findings.

\section{Introdução}

Considerando os anseios da sociedade do século XXI e as competências e habilidades desejadas no mercado de trabalho, a escola necessita mudar sua postura e envolver o estudante de forma ativa e atuante no seu processo de aprendizagem, bem como posicionar o professor no papel de orientador e mediador da discussão sobre a solução de problemas expostos. Para se envolver ativamente no processo de aprendizagem, o estudante deve ler, escrever, perguntar, discutir, além de realizar tarefas mentais de alto nível, como análise, síntese e avaliação, características intrínsecas da resolução de problemas e do desenvolvimento de projetos.

Vários indicadores tem mostrado que o Brasil não está conseguindo atingir metas traçadas em relação à educação, como pode ser visto nos resultados do IDEB 2015, bem como nas colocações alcançadas do último exame PISA, 59a posição em Leitura, 66a posição em Matemática e 63a posição em Ciências, entre os 70 países avaliados. Considerando os níveis globais de inovação 2016 (Dutta et al., 2016), o Brasil novamente ocupa uma posição periférica perante o mundo, perspectiva comprovada pela Confederação Nacional da Indústria (CNI), que atribui ao Brasil um nível de inovação muito baixo, resultado extraído através de um survey com profissionais e diretores de indústrias brasileiras.

Outra questão clara relacionada ao abismo cada vez maior entre o modelo educacional ainda vigente no Brasil, de cunho passivo, conteudista e centrado no professor, frente às expectativas de estudantes da geração $Z$ e alpha, bem como das demandas de um mercado de trabalho do século XXI, que exige inovação, criatividade, colaboração, pensamento crítico, liderança, maximização das interações sociais e culturais, entre outros, tem desaguado em altos índices de desmotivação e consequente evasão escolar, problema comprovado pelo Tribunal de Contas da União através de uma auditoria em 2012, que apresentou os diferentes graus de evasão nas diferentes modalidades em ensino dos Institutos Federais de Educação e Tecnologia. 
No relatório apresentado, foi verificada uma taxa de conclusão de $42,8 \%$ nos cursos superiores de tecnologia. Segundo o relatório, esse número poderia ser pior não fossem iniciativas isoladas de determinados campi no combate a evasão e aperfeiçoamento de instrumentos voltados ao acompanhamento periódico da elevação gradual da taxa de conclusão dos cursos. Esse número é muito distante da meta de $90 \%$ para a taxa de conclusão prevista no Projeto de Lei do Plano Nacional de Educação 2011-2020, ou mesmo da taxa de $80 \%$ para todas as modalidades de cursos ofertados pelos Institutos prevista no Termo de Acordo de Metas. O relatório aponta ainda que os altos índices de evasão nos cursos superiores de tecnologia são equivalentes aos índices encontrados em Universidades, Faculdades e Centros Universitários.

Utilizando dados do Sistema Gerenciador Acadêmico (SGA) do Instituto Federal de Educação, Ciência e Tecnologia da Paraíba (IFPB), foi pesquisada a quantidade de alunos que concluíram cursos de tecnologia no Campus João Pessoa no período de 2013.1 à 2015.1. Para verificar o grau de precisão das informações contidas no SGA foi realizada uma conferência minuciosa e individualizada dos concluintes do Curso Superior de Tecnologia em Sistemas para Internet e Redes de Computadores junto às coordenação do curso, comprovando os dados coletados. Os índices foram sintetizados na Tabela 1.

Tabela I. Quantidade de alunos que concluíram Cursos Superiores e Tecnologia

\begin{tabular}{|c|c|c|c|c|c|}
\hline & 2013.1 & 2013.2 & 2014.1 & 2014.2 & 2015.1 \\
\hline Sistemas para Internet & 9 & 6 & II & 9 & 6 \\
\hline Redes de Computadores & 15 & II & 13 & 4 & 7 \\
\hline Geoprocessamento & 5 & 6 & I & 8 & 3 \\
\hline Gestão Ambiental & 2 & 7 & 13 & 10 & 7 \\
\hline Telecomunicações & 10 & 3 & 4 & 0 & 4 \\
\hline Construção de Edifícios & 0 & 16 & 0 & 17 & 18 \\
\hline Automação Industrial & 4 & 0 & 2 & I & 5 \\
\hline Design de Interiores & 11 & 15 & 24 & 4 & 13 \\
\hline Negócios Imobiliários & 5 & 9 & 5 & 5 & 6 \\
\hline
\end{tabular}


Considerando a quantidade de estudantes que ingressam nos Cursos de Tecnologia do IFPB, a Tabela 2 apresenta a razão entre a quantidade de estudantes que concluem cada curso e a quantidade de alunos que ingressam em cada curso de graduação tecnológica. Como se observa, o fenômeno da evasão é preocupante e alarmante na grande maioria dos cursos analisados.

Tabela 2. Razão entre a quantidade de estudantes que concluem pela quantidade de alunos que ingressam em cursos superiores de tecnologia por período

\begin{tabular}{|cccccc} 
& 2013.1 & 2013.2 & 2014.1 & 2014.2 & 2015.1 \\
\hline Sistemas para Internet & $22,50 \%$ & $15 \%$ & $27,50 \%$ & 22,50 & $15 \%$ \\
\hline Redes de Computadores & $37,50 \%$ & $27,50 \%$ & $32,50 \%$ & $10 \%$ & $17,50 \%$ \\
\hline Geoprocessamento & $20 \%$ & $24 \%$ & $4 \%$ & $32 \%$ & $12 \%$ \\
Gestão Ambiental & $6,67 \%$ & $23,33 \%$ & $43,33 \%$ & $33,33 \%$ & $23,33 \%$ \\
Telecomunicações & $28,57 \%$ & $8,57 \%$ & $11,43 \%$ & $0 \%$ & $11,43 \%$ \\
\hline Construção de Edifícios & $0 \%$ & $40 \%$ & $0 \%$ & $42,50 \%$ & $45 \%$ \\
\hline Automação Industrial & $13,33 \%$ & $0 \%$ & $6,67 \%$ & $3,33 \%$ & $16,67 \%$ \\
\hline Design de Interiores & $36,67 \%$ & $50 \%$ & $80 \%$ & $13,33 \%$ & $43,33 \%$ \\
\hline Negócios Imobiliários & $16,67 \%$ & $30 \%$ & $16,67 \%$ & $16,67 \%$ & $20 \%$
\end{tabular}

A intensa experiência vivenciada durantes os três meses na Finlândia, fruto da seleção na chamada Professores para o Futuro 26/2015 SETEC/ $\mathrm{MEC} / \mathrm{CNPq}$, associada às pesquisas e visão prática observada na Finlândia sobre o que eu vinha desenvolvendo desde o doutorado, nas áreas de aprendizagem colaborativaehíbrida, bem comode tecnologiaseducacionais, proporcionou a mim uma mudança de postura como professor, além de despertar o interesse em disseminar esses conhecimentos e experiências para os professores e gestores do IFPB, utilizando para isso diferentes estratégias, que estão descritas nesse artigo. As ações implementadas por mim e por professores colaboradores do IFPB e de fora dele, estão sendo direcionadas ao compartilhamento de conhecimentos e experiências acerca das metodologias de Aprendizagem Ativa, em especial a Aprendizagem baseada em Projetos. 


\subsection{Aprendizagem baseada em Projetos}

Segundo Prince (2004), a Aprendizagem Ativa pode ser definida como um conjunto de métodos e estratégias de ensino que enxergam os estudantes como protagonistas no processo de aprendizagem. Segundo Chandrasekaran (2013), o método de Aprendizagem baseada em Projetos (ABPr) é uma das mais indicadas abordagens de aprendizagem ativa para se alcançar as competências do século XXI, especialmente devido a possibilidade na integração de diferentes cursos, a abrangência das habilidades desenvolvidas, a possibilidade de aplicação em problemas reais e o desenvolvimento de processos de liderança. A aprendizagem baseada em Projetos trata de um método de aprendizagem centrado no estudante com um vasto suporte metodológico e alicerçado no aprender fazendo de John Dewey (1933), e embora conduza o curso ou parte dele através de um projeto, diferencia-se do simples uso de um projeto como parte da avaliação de uma disciplina.

Segundo Hao et al. (2016), a Aprendizagem baseada em Projetos é uma abordagens de aprendizagem muito eficaz no desenvolvimento de autoregulação de aprendizagem e engajamento de estudantes em torno de questões autênticas. Hadim e Esche (2002) sugerem a grande eficácia da aplicação da ABPr em cursos de tecnologia e engenharia, sendo responsável inclusive pela reformulação completa de currículos de cursos na Europa. Bilgin at al. (2015) realizou uma revisão sistemática da literatura sobre os efeitos da Aprendizagem baseada em Projetos em diferentes fenômenos de aprendizagem e concluiu que a ABPr contribui positivamente com o seu sucesso acadêmico de modo geral, com a aprendizagem significativa de estudantes da área de ciências, com a aprendizagem individualizada, com a melhora da motivação e engajamento dos estudantes, bem como com o processo de auto-regulação da aprendizagem formal e informal.

De acordo com Chandrasekaran (2013), a Aprendizagem baseada em Projetos provê aos estudantes a habilidade de desenvolver liderança, colaboração, cooperação, propriedade, técnicas de apresentação, escrita e apropriação de tecnologias. Os padrões de ouro de ABPr, segundo Larmer et al. (2015), guiam estudantes no desenvolvimento de soluções que incorporam os seguintes elementos essenciais de um projeto: problema desafiador, investigação sustentável, autenticidade, voz e escolha do estudante, reflexão, revisão e críticas, habilidades analíticas, resolução de problemas e produto público. Segundo Bell (2010), as habilidades 
desenvolvidas através da aplicação de ABPr estão em total consonância quanto ao desenvolvimento dos $4 C^{\prime}$ s das competências do século XXI: pensamento crítico, comunicação, colaboração e criatividade, suportados pelas tecnologias de informação.

Larmer et al. (2015) lista seis padrões de conteúdos de Aprendizagem baseada em Projetos no sentido de apoiar professores e designers instrucionais no planejamento e execução de um curso: (i) o projeto é uma unidade completa ou o maior veículo de ensino dentro de uma unidade; (ii) as tarefas são abertas e com prazos delimitados promovendo a escolha e voz dos estudantes; (iii) realizado de forma colaborativa como um time; (iv) professor como um facilitador, utilizando parte das aulas nesse processo; (v) o projeto inclui um processo de investigação sustentável e a criação de artefatos (produtos); e (iv) é autêntico para o mundo real e para a vida do estudante.

Pesquisadores e praticantes defendem que a base de aprendizagem social para a ABPr é uma vantagem em relação à outros métodos ou à própria ausência de métodos. Segundo Bender (2015), a eficácia dessa abordagem pode ser resumida segundo três aspectos: (i) um currículo elaborado em torno de problemas com ênfase em habilidades cognitivas e conhecimento; (ii) um ambiente de aprendizagem centrado no aluno, que utilize pequenos grupos, e uma aprendizagem ativa em que professores atuem como facilitadores; (iii) resultados dos alunos focados no desenvolvimento de habilidades, motivação e amor pela aprendizagem permanente.

Em uma era em que as mídias digitais permitem a comunicação instantânea e há disponibilidade de informações quase ilimitada na Internet, os defensores da aprendizagem baseada em projetos sugerem que produzir sentido a partir da grande quantidade virtual de informações caóticas é exatamente o tipo de construção do conhecimento que todo estudante no mundo de hoje precisa dominar.

Larmer et al. (2015) examinou os últimos vinte e cinco anos de pesquisas relacionados a $\mathrm{ABPr}$, com foco nas experiências de professores e estudantes, na relação com as concepções contemporâneas de aprendizagem e no impacto quanto à motivação e ao engajamento dos estudantes. No que se refere às experiências de professores e estudantes, o autor reportou que do ponto de vista do estudante, para alcançar resultados de aprendizagem satisfatórios, há a necessidade de um cuidadoso e estruturado planejamento do projeto com base nos conhecimentos e habilidades dos estudantes, além 
de um suporte e orientação eficiente por parte dos professores. Quanto aos professores, a efetividade do uso de ABPr é proporcional à experiência do professor com a abordagem, ao suporte de ferramentas e tecnologias e ao apoio recebido dos pares, diretores e mentores.

Ainda segundo Larmer et al. (2015), ABPr é alinhado com o pensamento corrente da natureza da aprendizagem humana e com as condições necessárias para ajudar estudantes na compreensão do que foi estudado, na retenção do que foi aprendido e como aplicar em novos contextos e situações, além da maximização da motivação, engajamento e interesse dos estudantes.

As ações que vem sendo coordenadas no IFPB estão inseridas na tríade de ensino, pesquisa e extensão. No ensino, a experiência na Finlândia proporcionou uma mudança significativa em como eu conduzo o processo ensino aprendizagem nos cursos aos quais eu sou o responsável, além da possibilidade de oferta de treinamentos à professores, gestores e estudantes em temáticas de metodologias ativas de aprendizagem, ferramentas colaborativas e sociais de apoio ao processo de aprendizagem e avaliação formativa e diagnóstica. Na pesquisa, há dois projetos em andamento, um primeiro que se trata do desenvolvimento da ferramenta PrBL Tool para planejamento, execução e avaliação de Aprendizagem baseada em Projetos e o segundo que busca analisar o uso de projetos pelos professores do IFPB sob a ótica das Habilidades doSéculo XXI e das premissas da Aprendizagem baseada em Projetos. No âmbito da extensão, está em andamento um projeto de construção e fortalecimento de uma Rede Colaborativa de Professores do Século XXI, composto por professores e gestores de instituições federais e de ONGs. Todas as ações em andamento são frutos de editais do IFPB.

\section{Experiências de Aprendizagem baseada em Projetos no IFPB}

No ano posterior à experiência na Finlândia, três iniciativas foram implementadas em disciplinas dos cursos superiores de Tecnologia em Sistemas para Internet e Redes Computadores, bem como um curso baseado na Metodologia de Aprendizagem baseada em Projetos foi ofertado para estudantes e professores de seis cursos superiores do IFPB. Nesta seção são descritos brevemente essas ações.

Curso de Gerenciamento Ágil de Projetos utilizando Aprendizagem baseada em Projetos 
A primeira ação, fruto do trabalho de desenvolvimento realizado por grupos de professores de diferentes Institutos Federais, consistiu de um curso de Gerenciamento Ágil de Projetos seguindo a metodologia de Aprendizagem baseado em Projetos. O curso foi planejado e executado em três institutos federais concomitantemente, a tabela 3 apresenta uma visão geral da instancia do curso no IFPB.

Tabela 3. Instancia do curso de Gerenciamento Ágil de Projetos utilizando a Abordagem de Aprendizagem baseada em Projetos aplicada no IFPB

\begin{tabular}{|c|c|}
\hline \multicolumn{2}{|c|}{ IF Paraíba } \\
\hline Coordenador & Francisco Petrônio Alencar de Medeiros \\
\hline Colaboradores & $\begin{array}{l}\text { Paulo Sérgio dos Santos Jr., Heremita Lira, } \\
\text { Nadja Rodrigues e Flávio Lopes. }\end{array}$ \\
\hline Número de estudantes & 39 \\
\hline Nível dos estudantes & $\begin{array}{l}\text { Tecnologia em Sistemas para Internet, Redes } \\
\text { de Computadores, Geoprocessamento, } \\
\text { Construção de Edifícios e Gestão Ambiental. } \\
\text { Bacharelado em Engenharia Elétrica e } \\
\text { Administração. }\end{array}$ \\
\hline Duração do curso & From Oct 4th 2016 to Nov 29th 2016 \\
\hline Grupos & 7 groups \\
\hline Métodos Ativos de Aprendizagem & $\begin{array}{l}\text { Aprendizagem baseada em Projetos, Sala de } \\
\text { aula Invertida, Gamification e world cafe. }\end{array}$ \\
\hline \multicolumn{2}{|c|}{$\begin{array}{l}\text { A estrutura metodológica para a implementação do curso seguiu um } \\
\text { o de desenvolvimento de um projeto real. Começando com o "Warm } \\
\text { onde os estudantes identificaram as habilidades individuais de cada } \\
\text { mbro do grupo e a cultura do trabalho em times. Nesta etapa, eles } \\
\text { nheceram os objetivos e definiram o problema a ser resolvido. Na fase } \\
\text { "Inception", os estudantes foram estimulados a entender o problema a ser } \\
\text { lvido, usando o modelo Project Model Canvas (PMC). Durante a etapa } \\
\text { construção, "Build", os alunos começaram a trabalhar na construção } \\
\text { iva do projeto utilizando a metodologia de gerenciamento de projetos } \\
\text { RUM. }\end{array}$} \\
\hline
\end{tabular}


O ciclo terminou com a fase de "Post-morten" que representou uma discussão final sobre o projeto, considerando os sucessos, insucessos e problemas encontrado pelos times ao longo do desenvolvimento. Durante as quatro etapas, os estudantes foram estimulados a refletir sobre $o$ gerenciamento do projeto. Além disso, as atividades em grupo de tutoria, reuniões de coaching e o desenvolvimento do diário de bordo, compõem uma base de avaliação formativa e diagnóstica do trabalho desenvolvido. Os estudantes apresentaram os produtos através de uma solenidade pública no IFPB e publicizaram seus projetos em páginas Web ou do Facebook.

Os participantes do curso formaram times multidisciplinares para desenvolvimento dos projetos. Através de métodos ativos de aprendizagem, como a metodologia de Aprendizagem baseada em Projetos e Sala de Aula invertida, os participantes sentiram-se motivados e engajados devido ao caráter prático do curso e também por causa dos problemas reais escolhidos e desenvolvidos por cada um dos sete times. Cada time contou com um professor no papel de coach, responsável pela orientação dos projetos através de reuniões presenciais e a distância.

O grupo 1, formado por estudantes de Gestão Ambiental e Administração, desenvolveram um método para o reuso de óleo de cozinha. O grupo 2, formado por estudantes de Administração e Engenharia Elétrica, desenvolveram padrões de rotinas administrativas para a Unidade Acadêmica de Gestão. O grupo 3, formado por estudantes de Sistemas para Internet, desenvolveram uma plataforma colaborativa para Boas Práticas em Engenharia de Software. O grupo 4, formado por estudantes de Engenharia Elétrica, planejaram um curso com foco em colaboração e tecnologias para o ensino de Eletrônica para estudantes de ensino fundamental e médio. O grupo 5, formado por estudantes de Redes de Computadores, Geoprocessamento e Administração, desenvolveram os protótipos para dispositivos móveis de um mapa interativo para o IFPB. O grupo 6, formado por estudantes de Engenharia Elétrica e Sistemas para Internet, criaram uma sede da ACM (Association for Computing Machinery) no IFPB e o grupo 7, formado por estudantes de Engenharia Elétrica e Construção de Edifícios, desenvolveram um modelo de energia solar para residências. 
Metodologias de Aprendizagem Ativa em disciplinas dos cursos superiores de Informática

A disciplina de Programação Orientada a Objetos do curso de Redes de Computadores foi reformulada seguindo a Metodologia de Aprendizagem baseada em Projetos, segundo Larmer (2015): (i) o projeto guiou toda a disciplina, sendo o maior veículo de ensino; (ii) as tarefas foram abertas e com prazos delimitados promovendo a escolha e voz dos estudantes; (iii) realizado de forma colaborativa em times; (iv) professor como um facilitador, utilizando parte das aulas nesse processo; (v) o projeto incluiu um processo de investigação sustentável e a criação de artefatos (produtos); e (iv) foi autêntico para o mundo real e para a vida do estudante.

Todos os estudantes elaboraram um jornal de aprendizagem onde escreveram postagens semanalmente sobre dificuldades, aprendizados, considerações sobre o trabalho em equipe, entre outros. Considerando os produtos apresentados, os jornais de aprendizagem, o aprofundamento que os estudantes alcançaram no conteúdo da disciplina e a comparação entre as turmas dessa disciplina de períodos anteriores, comprovou-se que a aprendizagem baseada em projetos é muito indicada, proporcionando resultados muito satisfatórios e certas vezes até surpreendentes. Alguns ajustes serão implementados nos semestres futuros com o objetivo de obter resultados ainda melhores, sempre focando no estudante. A figura 1 apresenta um resumo do planejamento do projeto no período 2016.2 seguindo os templates da bie.org. 


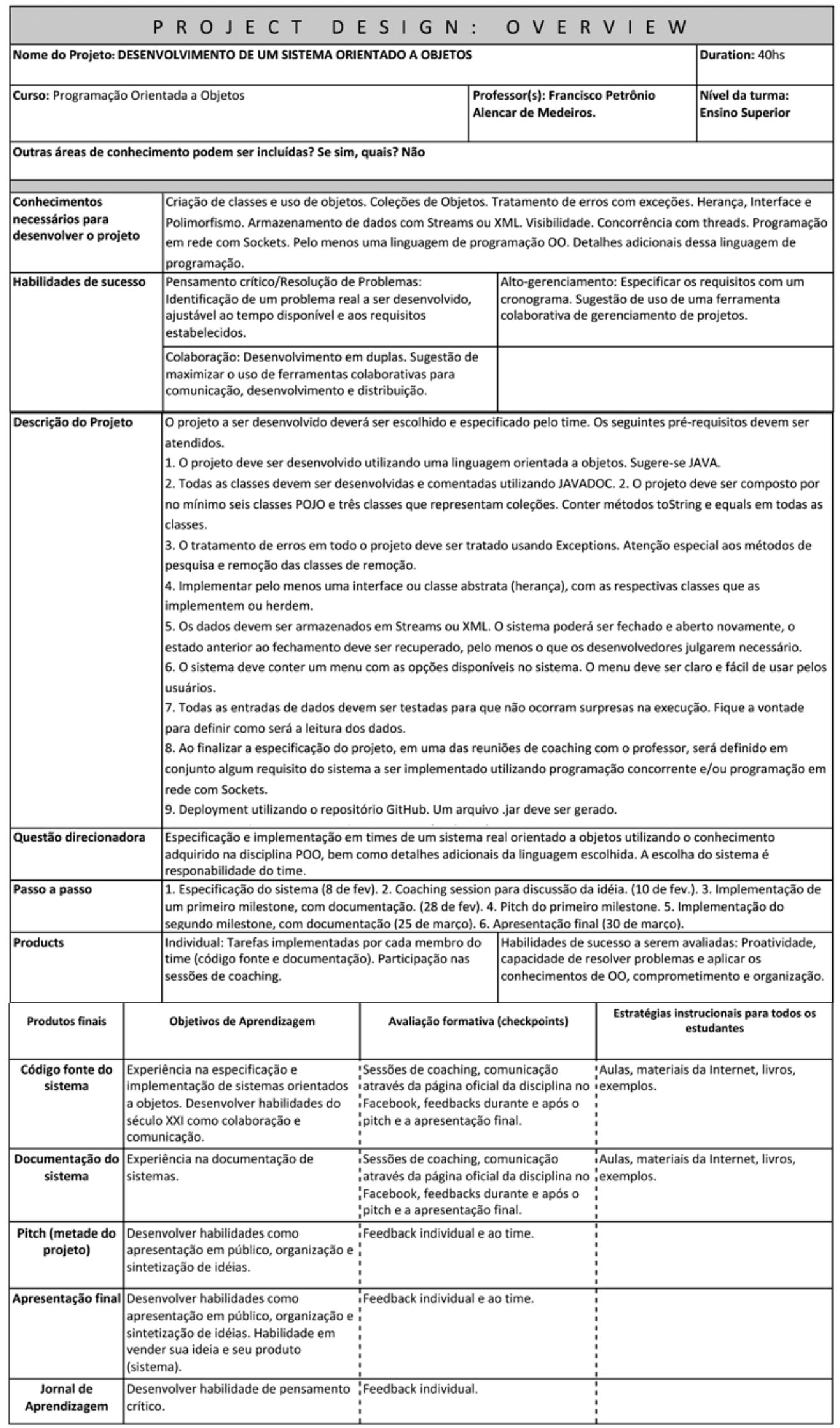

Figure I. Planejamento do Projeto da disciplina Programação Orientada a Objetos 
Na disciplina de Padrões de Projeto de Software do curso de Sistemas para Internet estão sendo aplicadas algumas metodologias ativas de aprendizagem, entre as quais Sala de Aula Invertida, bem como algumas estratégias e técnicas de compartilhamento de pensamentos, tais como brainstorming, brainwriting, world café e seminários. Por se tratar de uma disciplina avançada de programação e arquitetura de software, a Sala de Aula Invertida tem se apresentado como um método muito eficiente por permitir que na maior parte do tempo da aula os estudantes codifiquem, favorecendo portanto o aprender fazendo ou learning by doing, o que contribui para a memorização dos padrões de projeto e direcione o foco às suas aplicabilidades.

\section{Projeto de Extensão: Rede Colaborativa de Educadores do Século XXI}

O objetivo geral deste projeto é a criação e a formação de uma rede colaborativa e interdisciplinar de educadores do século XXI por meio da disseminação de métodos e práticas de aprendizagem ativa e centradas no estudante. Esta rede é formada por professores e gestores de instituições de ensino e organizações não governamentais espalhadas por todo o país. Como objetivos específicos, esse projeto almeja:

- Criar mecanismos para crescimento da rede por meio de palestras e cursos para professores, gestores e estudantes.

- Organização de repositórios de boas práticas de aprendizagem ativa.

- Integração no processo de ensino e aprendizagem estratégias e ferramentas ágeis de gerenciamento de projetos que podem ser utilizadas tanto para gerenciamento de disciplinas quanto para projetos desenvolvidos utilizando Aprendizagem baseada em Projetos.

- Integração de professores e estudantes, especialmente de licenciatura, de instituições de ensino superior, técnico, médio e fundamental, de diferentes autarquias e da iniciativa privada, bem como do terceiro setor.

- Contribuir para a formação de estudantes e profissionais com pensamento crítico e adaptados às habilidades do século XXI. 


\section{PrBL Tool: Uma ferramenta para o planejamento, execução e avaliação de Aprendizagem baseada em Projetos}

Muito embora as pesquisas e os relatos de experiências apresentem o quão promissora e efetiva é a Abordagem baseada em Projetos, autores dos livros mais referenciados sobre o tema, Bender (2015) e Larmer et al. (2015), bem como a mundialmente respeitada organização Buck Institute of Education, reportam minuciosamente os requisitos, as etapas e os padrões de ouro para o planejamento e o gerenciamento de iniciativas seguindo a $\mathrm{ABPr}$, deixando muito claro que a aplicação desse método vai muito além do simples uso de projetos em cursos, algo corriqueiro, porém não comprovadamente eficaz, em diferentes áreas do conhecimento.

Por outro lado, embora haja um consenso na literatura sobre o que caracteriza a Aprendizagem baseada em Projetos, há somente uma ferramenta que dê suporte ao complexo processo que é o planejamento de projetos segundo essa abordagem. A ferramenta em questão é o Project Planner (www.bie.org/project_planner) do Buck Institute of Education, que embora apresente um avanço em relação aos templates (modelos na forma de planilhas) existentes previamente e ainda muito utilizados, apresenta muitas limitações no que tange a abrangência do planejamento e gerenciamento de projetos, entre as quais: (i) específica para o planejamento de projetos com foco em ensino fundamental e médio (K12); (ii) impossibilidade de elaboração de um cronograma das tarefas planejadas e produtos gerados nos projetos; (iii) publicação do projeto para distribuição aos alunos exclusivo no portal bie.org; (iv) interface, documentação e ajuda exclusivas na língua inglesa e (v) não suporte ao planejamento das rubricas de avaliação, etapa imprescindível da Abordagem baseada em Projetos.

Há indícios de que esse projeto de pesquisa pode contribuir cientificamente na investigação de abordagens de aprendizagem ativa, bem como intervir no sentido de fortalecer a prática de Aprendizagem baseada em Projetos, ainda tão insipiente no Brasil. Enxerga-se também uma importante contribuição na área educacional, ao oferecer uma completa ferramenta que deverá ser útil tanto no suporte aos professores praticantes de $\mathrm{ABPr}$, quanto como um guia para professores que utilizam projetos em sala de aula, mas não usufruem do formalismo, da completude, da interdisciplinaridade e do caráter metodológico e científico da abordagem ABPr.

O objetivo geral deste trabalho consiste na investigação conceitual dos elementos essenciais do domínio da Aprendizagem baseada em Projetos como requisito para a especificação e implementação de uma ferramenta 
Web e Mobile de suporte ao planejamento, execução e avaliação de projetos, segundo ABPr, para todos os níveis educacionais. Esse projeto tem como objetivos específicos:

- Análise crítica e minuciosa da ferramenta Project Planner, identificando todas as limitações e omissões quanto aos elementos chaves da Aprendizagem baseada em Projetos.

- Análise de competidores quanto às linguagens de programação e arquiteturas de softwares para definição da plataforma que melhor se adapte aos requisitos funcionais e não funcionais da ferramenta a ser desenvolvida.

- Avaliação da solução proposta através de grupos focais conduzidos com professores do IFPB e da TAMK (Tampere University of Applied Sciences).

\section{Uso de Projetos em salas de aulas do IFPB: uma análise sob a ótica da Aprendizagem baseada em Projetos e das competências do Século XXI}

Embora difícil de comprovar quantitativamente, há cada vez mais professores utilizando o instrumento de projeto como parte da avaliação de disciplinas, comportamento amplamente observado também no espaço amostral do IFPB e de outros institutos federais de todo o Brasil. Por outro lado, através de observações e discussões com professores que pesquisam na área de Aprendizagem Ativa, há indícios de que as etapas de planejamento, execução, acompanhamento e avaliação dos projetos não são consideradas na maioria das experiências de uso de projetos em sala de aula, e principalmente, que os projetos são considerados como um mero apêndice da disciplina ou da avaliação da mesma, ferindo os princípios básicos da metodologia Aprendizagem baseada em Projetos, como reporta Larmer et al. (2015): “[...] quando você está planejando um projeto, tenha em mente que aprendizagem baseado em projetos não é a mesma coisa que 'fazer um projeto'. Para ser considerado ABPr, o método de instrução do curso baseia-se no projeto, além dele ser o veículo primário para abordar os padrões de conteúdo do curso. [...]"

Os relatos de experiência exitosos, assim como as pesquisas conduzidas nas duas últimas décadas, mostram o enorme potencial da metodologia ABPr em cursos de todos os níveis educacionais. Necessita-se identificar e impulsionar as estratégias de uso de projetos nos cursos do IFPB, e 
principalmente investigar mecanismos para transformar essas estratégias ad-hoc em iniciativas fundamentadas e efetivas de ABPr a serem espalhadas por todos os campi, oferecendo aos estudantes e professores todos os ganhos oriundos dessa metodologia ativa de aprendizagem.

O objetivo geral desse trabalho é investigar a utilização de projetos em disciplinas de cursos superiores do IFPB tomando como referência as etapas preconizadas na metodologia de Aprendizagem baseada em Projetos, bem como nos $4 C^{\prime}$ s das competências do século XXI, de modo a compilar um diagnóstico que aponte os gaps entre as práticas correntes da sala de aula e o método $\mathrm{ABPr}$, ensejando em um futuro próximo planejar estratégias de treinamento para diminuir esses gaps identificados. Como objetivos específico, têm-se:

- Revisão sistemática da literatura sobre Aprendizagem baseada em Projetos objetivando a elaboração de uma guia dos elementos essenciais no planejamento, execução e avaliação de projetos no âmbito de ABPr.

- Elaboração de três instrumentos de coleta de dados: survey (questionário), entrevista semiestruturada e um roteiro de observações diretas e indiretas para coleta de dados sobre o uso de projetos em sala de aula por professores do IFPB.

- Análise dos dados coletados utilizando a técnica de pesquisa qualitativa etnografia rápida, suportada pela ferramenta computacional de análise de dados qualitativos WebQDA.

\section{Conclusões}

A experiência proporcionada aos participantes da Chamada no 026-2015 da SETEC/MEC/CNPq foi extremamente valiosa e significativa. Falando em primeira pessoa, o treinamento e a vivência durante os três meses na Finlândia foram transformadores, principalmente no que tange ao papel de professor e o poder da educação em diferentes níveis educacionais. Considerando isso, percebeu-se como natural que os ensinamentos aprendidos e aperfeiçoados na Finlândia deveria ser disseminados com os professores do IFPB e porque não, alcançar outros educadores além dos muros do IFPB.

Em um primeiro momento, houve uma transformação nas disciplinas que eu venho ensinando há anos com ações que vão ao encontro da aprendizagem centrada no estudante. Como consequência, iniciou-se o 
projeto de extensão e os projetos de pesquisa voltados à metodologia de Aprendizagem baseada em Projetos, que por se tratar de uma metodologia ativa de aprendizagem ampla, propicia abarcar outros métodos e estratégias de aprendizagem ativa e em consonância às competências do século XXI.

Os projetos de extensão e pesquisa são frutos de editais públicos das pró-reitorias de extensão e pesquisa do IFPB. Na chamada Interconecta IFPB - $\mathrm{N}^{\circ}$ 01/2017 - Apoio a projetos de Pesquisa, Inovação, Desenvolvimento Tecnológico e Social, obtive-se um financiamento para taxa de bancada e pagamento de bolsas à estudantes no montante de $R \$ 9.600,00$, enquanto que no edital $N^{\circ}$ 06/2017/PRPIPG - Programa Institucional de Apoio à Pesquisa, Desenvolvimento Tecnológico e Inovação, estamos disputando um aporte de $\mathrm{R} \$ 10.200,00$ como taxa de bancada e pagamento de bolsas à estudantes de graduação.

\section{Referências Bibliográficas}

Bender, W. N. Aprendizagem baseada em projetos: educação diferenciada para o século XXI. Penso Editora, 2015.

Bilgin, I., Karakuyu, Y., \& Ay, Y. The effects of project based learning on undergraduate students' achievement and self-efficacy beliefs towards science teaching. Eurasia Journal of Mathematics, Science \& Technology Education, 11(3), 469-477, 2015.

Chandrasekaran, S., Stojcevski, A., Littlefair, G. and Joordens, M.. "Projectoriented design-based learning: aligning students' views with industry needs." International journal of engineering education 29, no. 5, 1109-1118, 2013.

Dutta, S.; Lanvin, B.; Wunsch-Vicent, S. (Ed.). The Global Innovation Index Report 2016: Winning with Global Innovation. 9. ed. Ithaca, Fontainebleau And Geneva: World Intellectual Property Organization (wipo), 2016.

Dewey, J. Having an experience. Art as experience, 36-59, 1934.

Hadim, H. A., \& Esche, S. K. Enhancing the engineering curriculum through project-based learning. In Frontiers in Education, 2002. FIE 2002. 32nd Annual (Vol. 2, pp. F3F-F3F). IEEE, 2002.

Hao, Q., Branch, R. M., \& Jensen, L. The effect of precommitment on student achievement within a Technology-rich Project-based learning Environment. TechTrends, 442-448, 2016.

Larmer, J., Mergendoller, J., \& Boss, S. Setting the standard for project based learning. ASCD, 2015.

Prince, M. Does active learning work? A review of the research. Journal of engineering education, 93(3), 223-231, 2004.

TCU - Tribunal de Contas da União. É excessiva a evasão dos cursos superiores ofertados pelos institutos federais de educação profissional e tecnológica? Revista do Tribunal de Contas da União, 2012. 